Revisão

\title{
Estado do conhecimento da produção acadêmica sobre o ensino e a aprendizagem de Educação Estatística no Ensino Superior
}

\author{
State of Knowledge of the academic production on teaching and learning of Statistic \\ Education in the Higher Education
}

\section{Estado del conocimiento de la producción académica sobre la enseñanza y el aprendizaje de educación estadística en la enseñanza superior}

Reinaldo Feio Lima ${ }^{1}$

[0000-0003-2038-7997]

\begin{abstract}
Resumo
Neste artigo, é apresentado um estudo de natureza qualitativa, do tipo Estado do Conhecimento, que teve como objetivo mapear a produção acadêmica sobre o ensino e a aprendizagem de Educação Estatística no Ensino Superior publicada nos anais do Encontro Nacional de Educação Matemática (ENEM) e do Seminário Internacional de Educação Matemática (SIPEM). Na análise de conteúdo dos 11 trabalhos selecionados, consideraram-se quatro categorias, a saber: (i) autor(es) e origem dos trabalhos; (ii) referencial teórico para embasamento; (iii) método e instrumentos de produção de dados; (iv) Tendências das pesquisas em Educação Estatística. A partir dos trabalhos analisados, é possível afirmar que o ensino e a aprendizagem de Educação Estatística no Ensino Superior presentes nesses trabalhos baseiam-se na perspectiva da implementação de práticas pedagógicas estatísticas, isto é, se possibilita a articulação entre teoria e prática dos fundamentos da Educação Estatística por meio de estratégias/atividades pedagógicas, sala de aula invertida fazendo uso de recursos tecnológicos, modelagem matemática como metodologia para o desenvolvimento da Educação Estatística, pesquisas que discutiram a formação de professores em grupos colaborativos. Também foi abordado o currículo do curso de Licenciatura em Matemática e Pedagogia.
\end{abstract}

Palavras-chave: Estado do Conhecimento. Educação Estatística. Ensino Superior.

\begin{abstract}
In this article is presented a qualitative study of the State of Knowledge, which had as goal to map the academic production on teaching and learning of Statistic in the Higher Education published in the annals of the National Meeting on Mathematical Education (NMME) and the International Seminary of Mathematical Education (ISME). In the analysis of content of the 11 selected works it was considered four categories, namely: (i) author(s) and origin of the works; (ii) theoretical referential for support; (iii) method and instruments of data production; (iv) Research trends in Statistical Education. From the analyzed works, it is possible to affirm that the teaching and learning of Statistic Education on the Higher Education present in these works are based on the perspective of implementation of statistics pedagogical practices, that is, it is enabled the articulation between theory and practice of the Statistic Education fundaments through strategies/pedagogical activities, inverted classroom using technological resources, mathematical modeling as methodology for the development of the Statistic Education, researches which discussed the formation of teachers in collaborative groups. It was also addressed the curriculum of the course of Graduation in Mathematic and Pedagogy.
\end{abstract} ${ }^{1}$ reinaldo.lima@unifesspa.edu.br, Doutor em Educação, Professor no Instituto de Engenharia do Araguaia (IEA),
Universidade Federal do Sul e Sudeste do Pará (UNIFESSPA), Marabá/Pará/Brasil. 
Keywords: State of the Knowledge. Statistical Education. Higher Education.

\section{Resumen}

En este artículo, se presenta un estudio de naturaleza cualitativa, del tipo Estado del Conocimiento, que tuvo como objetivo mapear la producción académica sobre la enseñanza y el aprendizaje de Educación Estadística en la Enseñanza Superior publicada en los anales del Encuentro Nacional de la Educación Matemática (ENEM) y del Seminario Internacional de Educación Matemática (SIPEM). En el análisis del contenido de los 11 trabajos seleccionados, se consideraron cuatro categorías, a continuación: (i) autor(es) y origen de los trabajos; (ii) referencial teórico para la base; (iii) método e instrumentos de producción de datos; (iv) Tendencias de la investigación en Educación Estadística. A partir de los trabajos analizados, es posible afirmar que la enseñanza y el aprendizaje de Educación Estadística en la Enseñanza Superior presentes en estos trabajos se fundamentan en la perceptiva de la implementación de prácticas pedagógicas estadísticas, esto es, se posibilita la articulación entre teoría y práctica de los fundamentos de la Educación Estadísticas por medio de estrategias/actividades pedagógicas, sala de clase invertida haciendo uso de recursos tecnológicos, modelaje matemática como metodología para el desarrollo de la Educación Estadística, pesquisas que discutieron la formación de profesores en grupos colaborativos. También fue abordado el currículo del curso de Licenciatura en Matemática y Pedagogía.

Palabras-llave: Estado del Conocimiento. Educación Estadística. Enseñanza Superior.

\section{Apresentando a temática}

O interesse pela pesquisa surgiu tanto a partir da participação do autor deste artigo nas discussões durante as duas últimas edições de dois principais eventos na Educação Matemática: o ENEM - Encontro Nacional de Educação Matemática - e o SIPEM - Seminário Internacional de Pesquisa em Educação Matemática -, ambos organizados pela Sociedade Brasileira de Educação Matemática (SBEM), bem como de inquietações sobre as pesquisas que vêm sendo desenvolvidas no Ensino Superior com enfoque na Educação Estatística.

Compreendemos Educação Estatística como uma subárea de estudo que se dedica a realizar pesquisas sobre os processos de ensino e de aprendizagem da Estatística, Combinatória e Probabilidade na Educação Básica e no Ensino Superior. Por essa razão, se configura como uma intersecção entre as duas áreas de conhecimento: a Educação e a Estatística.

No Brasil, o conteúdo de Estatística foi inserido na área da Matemática com a publicação dos Parâmetros Curriculares Nacionais (PCN), direcionados para os Anos Iniciais e Finais do Ensino Fundamental (BRASIL, 1997; 1998) e, depois, alcançando os estudantes do Ensino Médio (BRASIL, 2002; 2006). Mais recentemente, com a aprovação da Base Nacional Comum Curricular (BNCC), fazendo com que a Combinatória, a Estatística e a Probabilidade sejam parte de uma das cinco unidades de conhecimento da Matemática (BRASIL, 2018).

De acordo com as informações do sítio da SBEM,

os pesquisadores do GT12 atuam na área de Educação Estatística, que tem como objetivo estudar e compreender como as pessoas ensinam e aprendem Estatística, o que envolve os aspectos cognitivos e afetivos do ensinoaprendizagem, além da epistemologia dos conceitos estatísticos e o desenvolvimento de métodos e materiais de ensino etc., visando ao desenvolvimento do letramento estatístico. Para tal, a Educação Estatística 
utiliza-se de recursos teórico-metodológicos de outras áreas, como Educação Matemática, Psicologia, Pedagogia, Filosofia e Matemática, além da própria Estatística.

Por ser uma área de estudo recente, que ainda está se consolidando, assim como a Educação Matemática, em geral, as pesquisas sobre o raciocínio e a aprendizagem em Estatística, Combinatória e Probabilidade, atreladas às reflexões sobre o currículo de Matemática, surgiram, segundo Lopes (2008), nos primeiros anos do século XXI. Santos (2014, p. 3) argumenta que a inserção da Educação Estatística nos meios acadêmicos brasileiros "foi lenta e tardia... isto, em parte, se deve ao fato de ser a Estatística (assim como a própria Matemática) uma ciência preterida em favor de estudos literários e jurídicos, considerados de maior prestígio e tradição erudita". Recentemente, a promulgação da Base Nacional Comum Curricular (BNCC) trouxe um grande avanço para o processo de ensino e de aprendizagem da Estatística, Combinatória e da Probabilidade ao propor que esses temas sejam trabalhados desde os primeiros anos de escolaridade.

O Encontro Nacional de Educação Matemática (ENEM), criado pela SBEM, é considerado importante no âmbito nacional, porque congrega o universo dos segmentos envolvidos com a Educação Matemática: professores da Educação Básica, professores e estudantes das Licenciaturas em Matemática e em Pedagogia, estudantes da Pós-Graduação e pesquisadores. A cada encontro, constatamos o interesse pelas discussões sobre a Educação Matemática, seus fazeres múltiplos e complexos, tendências metodológicas e pesquisas que constituem a área.

O XIII ENEM aconteceu em 2019, em Cuiabá, no Mato Grosso. O tema desse encontro foi "Educação Matemática com as Escolas da Educação Básica: interfaces entre pesquisas e sala de aula". Esse encontro foi composto por 25 Subeixos, a saber:

$>1$ - Avaliação em Educação Matemática;

$>2$ - Desenvolvimento curricular em Educação Matemática;

$>3$ - Recursos Didáticos para Educação Matemática na Infância;

$>4$ - Recursos Didáticos para Educação Matemática nos Anos Finais do Ensino Fundamental, no Médio e no Superior;

$>5$ - Práticas inclusivas em Educação Matemática;

> 6 - Educação Matemática de jovens e adultos;

$>7$ - Resolução de problemas e investigações matemáticas;

$>8$ - Modelagem em Educação Matemática;

$>9$ - Etnomatemática;

> 10 - O papel e o uso de tecnologias digitais no ensino e na aprendizagem matemática;

$>11$ - Gestão e Avaliação de Feiras de Matemática;

$>12$ - Educação Matemática e Diversidade Cultural;

$>13$ - Psicologia da Educação Matemática;

> 14 - Dimensões filosóficas, sociológicas e políticas na Educação Matemática;

$>15$ - Pesquisas em práticas escolares;

$>16$ - Pesquisas sobre o ensino e a aprendizagem de Matemática em nível superior;

> 17 - História da Educação Matemática;

> 18 - História da Matemática no processo de ensino e de aprendizagem;

> 19 - Tecnologias digitais em Educação Matemática; 
$>20$ - Aprendizagem docente e desenvolvimento profissional de professores que ensinam Matemática;

> 21 - Políticas públicas curriculares na formação de professores que ensinam Matemática;

$>22$ - Formação inicial de professores que ensinam Matemática;

$>23$ - Formação continuada de professores que ensinam Matemática;

$>24$ - A parceria universidade e escola na formação de professores que ensinam Matemática;

$>25$ - Conhecimento, prática e identidade do professor que ensina Matemática.

O Seminário Internacional de Pesquisa em Educação Matemática (SIPEM) foi idealizado e organizado periodicamente, de três em três anos, pela Sociedade Brasileira de Educação Matemática (SBEM). Além disso, o SIPEM propicia a formação de grupos integrados de pesquisa, ao congregar pesquisadores brasileiros e estrangeiros, o que possibilita o avanço das pesquisas em Educação Matemática em nosso País. Assim, são objetivos do SIPEM:

$>$ Promover o intercâmbio entre os grupos que, em diferentes países, se dedicam às pesquisas cujo tema é a Educação Matemática;

$>$ Divulgar as pesquisas brasileiras no âmbito da Educação Matemática;

$>$ Promover o encontro dos pesquisadores em Educação Matemática, proporcionandoIhes a possibilidade de conhecer as investigações que estão sendo realizadas na atualidade;

$>$ Propiciar a formação de grupos integrados de pesquisas que congreguem pesquisadores brasileiros e estrangeiros;

$>$ Possibilitar o avanço das pesquisas em Educação Matemática.

O VII SIPEM aconteceu em 2018 em Foz do Iguaçu, no Paraná. Esse seminário foi composto por 15 Grupos de Trabalho, a saber: GT 01 - Matemática na Educação Infantil e nos Anos Iniciais do Ensino Fundamental; GT 02 - Educação Matemática nos Anos Finais do Ensino Fundamental e Ensino Médio; GT 03 - Currículo e Educação Matemática; GT 04 - Educação Matemática no Ensino Superior; GT 05 - História da Matemática e Cultura; GT 06 - Educação Matemática: novas tecnologias e Educação à distância; GT 07 - Formação de professores que ensinam Matemática; GT 08 - Avaliação em Educação Matemática; GT 09 - Processos cognitivos e linguísticos em Educação Matemática; GT 10 - Modelagem Matemática; GT 11 Filosofia da Educação Matemática; GT 12 - Ensino de Probabilidade e Estatística; GT 13 Diferença, Inclusão e Educação Matemática; GT 14 - Didática da Matemática e GT 15 - História da Educação Matemática.

Portanto, neste artigo, o objetivo é apresentar um Estado do Conhecimento sobre a produção acadêmica referente ao ensino e à aprendizagem de Educação Estatística no Ensino Superior, a partir dos trabalhos publicados no ENEM/2019 e do SIPEM/2018. Para isso, realizamos um Estado do Conhecimento que é caracterizado pela "identificação, registro, categorização que levem à reflexão e síntese sobre a produção científica de uma determinada área, em um determinado espaço de tempo, congregando periódicos, teses, dissertações e livros sobre uma temática específica" (MOROSINI; FERNANDES, 2014, p. 155).

Face ao exposto, apresenta-se, na sequência, o procedimento metodológico, assim como a caracterização do lócus considerado neste Estado do Conhecimento. Em seguida, nos resultados e discussões, são descritas e analisadas as categorias que sintetizaram a produção acadêmica acerca do ensino e aprendizagem de Educação Estatística no Ensino Superior. Nas conclusões e implicações, é apresentada uma síntese das análises expostas. 


\title{
2 Procedimentos metodológicos
}

Com o objetivo de mapear a produção acadêmica sobre o ensino e a aprendizagem de Educação Estatística no Ensino Superior, optou-se por estudar pesquisas acadêmicas brasileiras produzidas nos dois principais eventos na Educação Matemática: o ENEM Encontro Nacional de Educação Matemática - e o SIPEM - Seminário Internacional de Pesquisa em Educação Matemática. A pesquisa se constituiu pelo método qualitativo, pois nosso enfoque está relacionado à compreensão e discussão dos dados obtidos nos anais dos referidos eventos, uma vez que se busca problematizar, e não apenas apresentar os resultados obtidos nos trabalhos selecionados (BICUDO, 2014). A esse respeito, Creswell (2007, p. 186) afirma que a pesquisa qualitativa é fundamentalmente interpretativa, na qual o pesquisador faz uma interpretação dos dados, incluindo: "[...] o desenvolvimento da descrição de uma pessoa ou de um cenário, análise de dados para identificar temas ou categorias e, finalmente, fazer uma interpretação ou tirar conclusões sobre seu significado, pessoal e teoricamente".

De natureza bibliográfica, a qual "[...] tem como objetivo primordial a descrição das características de determinada população ou fenômeno ou, então, o estabelecimento de relações entre variáveis" (GIL, 2002, p. 42). A esse respeito, Gatti (2002, p. 11) enfatiza que, "quanto mais próximas estiverem as pesquisas das realidades e contextos de atuação dos pesquisadores, estes adquirirão diversos conhecimentos, na perspectiva de apontar possíveis soluções para os problemas que os afligem". Segundo Ferreira (2002), tais pesquisas são

\begin{abstract}
Definidas como de caráter bibliográfico, elas parecem trazer em comum o desafio de mapear e de discutir uma certa produção acadêmica em diferentes campos do conhecimento, tentando responder que aspectos e dimensões vêm sendo destacados e privilegiados em diferentes épocas e lugares, de que formas e em que condições têm sido produzidas certas dissertações de mestrado, teses de doutorado, publicações em periódicos e comunicações em anais de congressos e de seminários. Também são reconhecidas por realizarem uma metodologia de caráter inventariante e descritivo da produção acadêmica e científica sobre o tema que busca investigar, à luz de categorias e facetas que se caracterizam enquanto tais em cada trabalho e no conjunto deles, sob os quais o fenômeno passa a ser analisado." (p. 258, grifo nosso).
\end{abstract}

A busca nestas fontes de referência aconteceu no período que vai de fevereiro a março de 2020 e se deu, primeiramente, pela busca por descritores, que são eles: estatística, combinatória, probabilidade e Educação Estatística.

A segunda ação aconteceu com a leitura dos títulos, resumo e palavras-chave dos 11 trabalhos encontrados nesta busca anterior e, na maioria dos casos, pela leitura dos trabalhos em sua íntegra. Ao finalizar as buscas, havíamos selecionado um total de onze trabalhos, publicados nos anais da última edição do ENEM/2019 e SIPEM/2018, desenvolvidos no período de 2018 a 2019. No Quadro 1, apresentamos uma síntese da seleção realizada. 
Quadro 1 - Dados dos trabalhos selecionados

\begin{tabular}{|c|c|c|c|}
\hline Fonte & Título & Autor(es) & Instituição \\
\hline \multirow{6}{*}{ 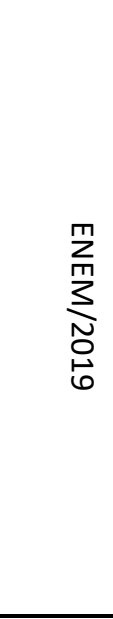 } & $\begin{array}{l}\text { Experiências no ensinar e no promover o aprender estatística } \\
\text { para estudantes do bacharelado em Biblioteconomia }\end{array}$ & $\begin{array}{l}\text { Silva e Samá } \\
(2019)\end{array}$ & FURG \\
\hline & $\begin{array}{l}\text { Contribuições do contexto colaborativo na formação docente } \\
\text { para o ensino de Estatística }\end{array}$ & \begin{tabular}{|l|} 
Schreiber e \\
Porciúncula (2019) \\
\end{tabular} & FURG \\
\hline & $\begin{array}{l}\text { Sala de aula invertida no ensino de Estatística: um estudo de } \\
\text { caso no curso de Administração }\end{array}$ & $\begin{array}{l}\text { Costa, Costa e } \\
\text { Carvalho (2019) }\end{array}$ & UFMT \\
\hline & $\begin{array}{l}\text { Estatística nas licenciaturas em Matemática no Estado de Mato } \\
\text { Grosso }\end{array}$ & $\begin{array}{l}\text { Rocha, Rodrigues, } \\
\text { Silva e Antunes } \\
\text { (2019) }\end{array}$ & UNEMAT \\
\hline & $\begin{array}{l}\text { Percepção de estudantes sobre uma abordagem para o ensino } \\
\text { de Estatística no contexto do ensino híbrido }\end{array}$ & $\begin{array}{l}\text { Andrade, Almeida } \\
\text { e Esquincalha } \\
\text { (2019) }\end{array}$ & UFRJ \\
\hline & $\begin{array}{l}\text { Competências estatísticas e o ensino na área de ciências } \\
\text { agrárias: uma experiência no curso de Zootecnia }\end{array}$ & Pereira (2019) & UFFPR \\
\hline \multirow{5}{*}{ 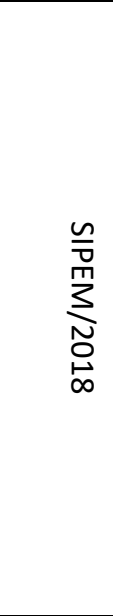 } & $\begin{array}{l}\text { A modelagem matemática e o letramento estatístico no ensino } \\
\text { de gráficos }\end{array}$ & $\begin{array}{l}\text { Campos e } \\
\text { Coutinho (2018) } \\
\end{array}$ & PUCSP \\
\hline & $\begin{array}{l}\text { As atitudes em relação à probabilidade e à estatística e o } \\
\text { desempenho acadêmico de alunos de um bacharelado em } \\
\text { Ciências e Tecnologia }\end{array}$ & $\begin{array}{l}\text { Júnior, Zamora e } \\
\text { Souza (2018) }\end{array}$ & UFABC \\
\hline & $\begin{array}{l}\text { Contribuições da modelagem matemática para o } \\
\text { desenvolvimento da literacia estatística: uma experiência em } \\
\text { um curso superior tecnológico }\end{array}$ & $\begin{array}{l}\text { Perim e } \\
\text { Wodewotzki } \\
(2018) \\
\end{array}$ & FTI UNESP \\
\hline & Desafios da Educação Estatística em cursos de Pedagogia & $\begin{array}{l}\text { Conti, Nunes e } \\
\text { Goulart (2018) } \\
\end{array}$ & $\begin{array}{l}\text { UFMG } \\
\text { UFRGS } \\
\text { IFSP }\end{array}$ \\
\hline & $\begin{array}{l}\text { Ensino de estatística: discussão sobre sequências didáticas } \\
\text { aplicadas por estudantes de licenciatura em Pedagogia em } \\
\text { ambiente virtual. }\end{array}$ & Figueiredo (2018) & UNIMES \\
\hline
\end{tabular}

Fonte: Dados da pesquisa.

A análise dos resumos ou mesmo dos trabalhos na íntegra foi realizada à luz da Análise de Conteúdo de Bardin (2016). Segundo a autora, a Análise de Conteúdo corresponde a um conjunto de técnicas de "análise das comunicações, visando obter, por procedimentos objetivos e sistemáticos de descrição do conteúdo das mensagens, indicadores (quantitativos ou não) que permitam a inferência de conhecimentos relativos às condições de produção/recepção destas mensagens" (BARDIN, 1977, p. 42), considerando as seguintes etapas:

1) Pré-análise, triagem e organização do material a ser pesquisado por meio de "leitura flutuante" (BARDIN, 2016) dos onze trabalhos selecionados para o corpus do presente artigo. Para Bardin (1977, p. 96), "o corpus é o conjunto dos documentos tidos em conta para serem submetidos aos procedimentos analíticos". O corpus da presente pesquisa são os trabalhos publicados e selecionados nos anais do ENEM/2019 e SIPEM/2018.

2) Exploração do material, que consiste nas operações de codificação, isto é, operações de recorte (trechos dos trabalhos analisados), agregação e enumeração das informações textuais que, posteriormente, constituem categorias a priori. Para a referida autora, 0 processo de categorização caracteriza-se como "uma operação de classificação de elementos 
constitutivos de um conjunto, por diferenciação e, seguidamente, por reagrupamento segundo gênero (analogia), com os critérios previamente definidos" (Bardin, 1977, p. 117).

3) Tratamento dos dados, realizou-se a análise das categorias a priori, interpretadas conforme embasamentos teóricos e conhecimentos já desenvolvidos em torno da temática Educação Estatística. Para Bardin (2011), esse é o momento da intuição/compreensão, da análise reflexiva e crítica, que terá como referência a discussão realizada pelos autores dos trabalhos selecionados (Quadro 1).

Para analisar os trabalhos selecionados, elaboramos, conforme indicado por Soares e Maciel (2000), uma categorização fazendo o levantamento dos seguintes aspectos:

C1 - autor(es) e origem dos trabalhos;

C2 - referencial teórico para embasamento;

C3 - método e instrumentos de produção de dados;

C4 - Educação Estatística na formação inicial de professores que ensinam Matemática.

Na próxima seção, apresentamos e discutiremos os dados por meio do procedimento da Análise de Conteúdo (BARDIN, 2016; 1977).

\section{Apresentação e discussão dos dados}

A categoria C1, "autor(es) e origem dos trabalhos", concerne sobre a autoria dos trabalhos e as instituições que os desenvolveram, fato evidenciado nos trabalhos analisados. 0 "Quadro 1" ilustra a presença da origem dos trabalhos e suas autorias e, conseguintemente, os Estados como: Mato Grosso, Minas Gerais, Paraná, Rio de Janeiro, Santana Catarina e São Paulo, os quais também se destacaram durante os anos cuja produção de pesquisas em Educação Estatística ocorreu no âmbito da Educação Matemática.

Além disso, observamos que grande parte dos autores está vinculada a instituições públicas de ensino, concentradas, principalmente, nas regiões Sul e Sudeste, por exemplo: Universidade Federal do Rio Grande (FURG); Universidade Federal de Mato Grosso (UFMT); Universidade do Estado do Mato Grosso (UNEMAT); Universidade Federal do Rio de Janeiro (UFRJ); Universidade Tecnológica Federal do Paraná (UTFPR); Universidade Federal do ABC (UFABC); Universidade Estadual Paulista (UNESP); Universidade Federal de Minas Gerais (UFMG); Universidade Federal do Rio Grande do Sul (UFRGS); Instituto Federal de Educação, Ciência e Tecnologia de São Paulo (IFSP). No entanto, houve também a emergência de instituições particulares, exemplificando, Pontifícia Universidade Católica de São Paulo (PUCSP); Faculdade de Tecnologia de Itapetininga (FTI) e Universidade Metropolitana de Santos (UNIMES). Isso nos leva inferir sobre a origem dos autores dos trabalhos, uma vez que não há um núcleo de pesquisa de destaque, os estudos apresentam-se diluídos, ou seja, a pesquisa é de abrangência nacional, estadual e regional.

Essas considerações ainda revelam que a Educação Estatística, como tendência na prática pedagógica e na pesquisa no Ensino Superior, é desenvolvida em parceria com diferentes instituições de ensino. Um exemplo disso é o trabalho intitulado "Desafios da Educação Estatística em cursos de Pedagogia", escrito por três autores de diferentes instituições: UFMG, UFRGS e IFSP. Outro ponto de destaque diz respeito ao fato de que a maioria dos trabalhos é produzida coletivamente, a grande parte deles em duplas ou trios. Isso nos leva a inferir que muitos sejam resultados de pesquisas acadêmicas, isto é, fruto de investigações de mestrandos e doutorados, ou até mesmo escritos por pesquisadores preocupados com a temática. Diante disso, 
Diversos grupos de pesquisa têm demonstrado interesse na Educação Estatística dentro do cenário brasileiro. Campos, Wodewotzki e Jacobini (2011) destacam, por exemplo, o Grupo de Estudos e Pesquisas em Educação Estatística-GEPEE, da UNICSUL-SP; o Grupo de Pesquisa em Educação Estatística-GPEE, da UNESP-Rio Claro; o Grupo de Prática Pedagógica em Matemática-PRAPEM e o Grupo de Psicologia e Educação MatemáticaPSIEM, ambos da UNICAMP; o Grupo de Pesquisa em Educação EstatísticaEduEst, da FURG-RS; o Grupo de Processo de Ensino-aprendizagem da Matemática no ensino básico - PEA-Mat, da PUC-SP; e o Grupo de Pesquisa em Educação Matemática, Estatística e Ciências-GPEMEC, da UESC-BA.

A criação desses grupos de pesquisas foi crucial para o crescimento da Educação Estatística no cenário brasileiro, aumentando as discussões por referenciais teóricos que sustentam os trabalhos a serem desenvolvidos. Essa ideia estabelece relação também com a categoria C2.

Na sequência, a categoria C2 - Sobre o referencial teórico para embasamento expressa alguns aspectos teóricos que embasaram a discussão sobre Educação Estatística no Ensino Superior. Dos 11 trabalhos analisados, apenas dois estudos assumiram um referencial teórico, a saber: Campos e Coutinho (2018) fundamentaram seu estudo no conceito de letramento estatístico presente nos fundamentos da Educação Estatística de Gal (2002) e Figueiredo (2018) investigou o conhecimento de Estatística durante prática em uma sala de aula.

No geral, os autores dos 11 trabalhos analisados embasam seus estudos em autores presentes na literatura nacional e internacional. Sendo assim, listamos os autores citados com mais frequência nos trabalhos apresentados no ENEM/2019 e SIPEM/2018: Batanero (2013), Borba (2010); Campos (2007); Wodewotzki; Jacobine (2011); Cazorla (2002); Damin (2018); Gal (2002), Lopes (2008; 2013); Porciúncula e Samá (2015); Silva (2018), Shulman (1986), Vendramini e Brito (2001); Viali (2007); Zieffler, Garfield e Fry, (2018); Wodewotzki e Jacobini (2004).

A categoria C3 - Sobre método e instrumentos de produção de dados expressa alguns aspectos relativos ao método e aos instrumentos de produção de dados assumidos ou não pelos autores dos trabalhos.

Nem todos os trabalhos apresentam o método de pesquisa assumido no resumo ou no decorrer do texto. Daqueles que explicitam, todos utilizam o método qualitativo (CRESWELL, 2010). Para a produção de dados, em geral, foram usados como procedimento: observação participante, gravação de áudio e vídeo, entrevistas, questionários, permitindo a materialidade e a interpretação do fenômeno estudado (DENZIN; LINCON, 2006). Como exemplos dessa variação, temos:

[...] O corpus de análise foi constituído pelas narrativas dos professores que participaram de quatro encontros, gravadas em áudio (e, posteriormente, transcritas), e os registros do diário de campo da pesquisadora (SCHREIBER; PORCIÚNCULA, 2019, p. 4). [...] Sendo assim, utilizamos a observação participante, a entrevista e o registro audiovisual (COSTA; COSTA; CARVALHO, 2019, p. 6). [...] Os procedimentos para coletar os dados foram observações de diálogos, debates e análises de produções dos alunos (CAMPOS; COUTINHO, 2018, p. 6). [...], as quais foram gravadas em áudio e, 
posteriormente, transcritas na íntegra pela professora/pesquisadora (PERIM; WODEWOTZKI, 2018, p. 5).

A predominância do método qualitativo, evidenciada nesta categoria, se justifica por admitir "um leque diversificado de procedimentos, sustentados por diferentes concepções de realidade e de conhecimento" (BICUDO, 2011, p. 24). Utilizamos, pois, uma diversidade de instrumentos para a produção de dados, como é mencionado na descrição desta categoria.

$O$ uso de diferentes instrumentos de produção de dados pelos autores dos trabalhos analisados "pode indicar que as pesquisas têm buscado compreender seus objetos de estudo de distintas maneiras, com o intuito de conhecer, com o auxílio de variados instrumentos, aquilo que se dedicaram a estudar" (KLÜBER; TAMBARUSSI, 2017, p. 570).

Tendo em vista a categoria C4, última categoria emergente deste artigo, intitulada, “ Tendências das pesquisas em Educação Estatística", a análise dos artigos selecionados neste Estado do Conhecimento possibilitou destacar seis tendências das pesquisas em Educação Estatística, a saber: a) Avaliação; b) Contexto colaborativo; c) Modelagem matemática; d) Currículo; e) Sala de aula invertida; e) Estratégia pedagógica.

Entre os artigos selecionados que discutiram avaliação, pode-se destacar a pesquisa desenvolvida por Júnior, Zamora e Souza (2018) intitulada "As atitudes em relação à probabilidade e à estatística e o desempenho acadêmico de alunos de um bacharelado em Ciências e Tecnologia". Para isso, foi utilizada Escala de Elena Auzmendi, que considera as atitudes em relação à Matemática e à Estatística, sendo composta por cinco fatores básicos (utilidade, ansiedade, confiança, prazer e motivação), a qual foi traduzida e adaptada ao português considerando as atitudes em relação à Probabilidade e à Estatística composta de 25 itens. Essa escala foi aplicada a 134 alunos. De maneira geral, os resultados iniciais mostram que eles não têm confiança na resolução de problemas estatísticos e probabilísticos; não é prazeroso pensar em elementos da Probabilidade e da Estatística; utilidade da Probabilidade e da Estatística no mercado de trabalho; e motivação em trabalhar com a Probabilidade e a Estatística em sala de aula na medida de sua utilidade no mercado de trabalho.

Schreiber e Porciúncula (2019) discutiram as contribuições do contexto colaborativo na formação docente para o ensino de Estatística. As autoras formaram um grupo colaborativo que se reúne mensalmente composto por professores do Ensino Fundamental, Ensino Médio e Ensino Superior, com o intuito de compartilhar, discutir e investigar a prática pedagógica na perspectiva do Letramento Estatístico. As narrativas realizadas pelos participantes do grupo colaborativo mostraram os primeiros movimentos relativos à mobilização de novas práticas pedagógicas para o ensino de Estatística a partir desse contexto colaborativo. Uma vez que grupos colaborativos funcionam como espaços "de produção e difusão de conhecimento e tornam-se um ambiente de excelência para uma contínua formação docente, pois tomam a prática do professor como ponto de partida", ou seja, onde "cada um contribui com o que pode e recebe ajuda naquilo de que necessita" (PENHA, 2013, p. 242). O contexto colaborativo na Educação Estatística com foco no Ensino Superior possibilita que sejam "criadas oportunidades para o professor explorar e questionar seus próprios saberes e práticas, bem como para conhecer saberes e práticas de outros professores, permitindo-lhe aprender por meio do desafio das próprias convicções" (FERREIRA, 2013, p. 152).

Uma terceira tendência, presente nas discussões relacionadas as pesquisas sobre Educação Estatística no Ensino Superior, abrangeu a modelagem matemática, apresentada em 
dois trabalhos. Campos e Coutinho (2018), no trabalho intitulado "A modelagem matemática e o letramento estatístico no ensino de gráfico", foi desenvolvido em uma turma de alunos da disciplina de Estatística de um curso de graduação em Ciências Econômicas de uma universidade privada da cidade de São Paulo, cujo objetivo foi discutir a estratégia didática da modelagem matemática para trabalhar o ensino e a aprendizagem de gráficos estatísticos. Já o trabalho de Perim e Wodewotzki (2018) - intitulado "Contribuições da modelagem matemática para o desenvolvimento da literacia estatística: uma experiência em um curso superior tecnológico" - analisou as contribuições do ambiente de modelagem matemática, em um curso superior tecnológico, para o desenvolvimento das competências de literacia, raciocínio e pensamento. Em ambos os trabalhos, os autores defendem a ideia de que a modelagem matemática se apresenta como um ambiente de aprendizagem favorável para o desenvolvimento do letramento estatístico e a literacia estatística, conforme apontado por Zieffler, Garfield e Fry, (2018). Sobre isso, modelagem matemática constitui-se, então, em uma estratégia didática compatível com todos os níveis escolares, que pode ser caracterizada como um ambiente de aprendizagem (a ser construído na sala de aula) em que os estudantes são convidados (pelo professor) para investigar, através da Matemática, situações extraídas do dia a dia ou mesmo de outras ciências (CAMPOS, 2016; (CAMPOS; WODEWOTZKI; JACOBINI, 2011).

Entre os artigos que discutiram o currículo, podem-se destacar os trabalhos desenvolvidos por Rocha, Rodrigues, Silva e Antunes (2019) e Conti, Nunes e Goulart (2018), que analisaram o currículo prescrito dos cursos de Licenciatura em Matemática e Pedagogia brasileiras, respectivamente.

Rocha, Rodrigues, Silva e Antunes (2019) analisaram como os Projetos Pedagógicos dos Cursos de Licenciatura em Matemática no Estado de Mato Grosso abordam a Estatística na Formação Inicial de Professores. Para isso, os pesquisadores realizaram uma pesquisa documental com 11 Projetos Pedagógicos de Cursos. Para analisar os dados, utilizaram-se os procedimentos da Análise de Conteúdo na perspectiva elucidada por Bardin. Considerando a matriz curricular e as ementas das disciplinas oferecidas nestes cursos, foi permitido compreender que os conhecimentos de Estatística na formação inicial de professores precisam de um redirecionamento para a Educação Estatística que o futuro professor de Matemática desenvolverá nas aulas do Ensino Fundamental e Médio. Já os estudos desenvolvidos por Conti, Nunes e Goulart (2018) realizaram um levantamento dos projetos pedagógicos, matrizes curriculares e súmulas/ementas de Instituições Estaduais, Federais e Municipais (públicas) da Região Sul do Brasil que ofertam esse curso de Pedagogia. Para se produzir os resultados dessa análise de documentos foi utilizada a estatística descritiva, através do software SPSS versão 18.0. Os resultados indicaram que, das instituições da Região Sul, 17 (42,5\%) estão localizadas em Santa Catarina, 16 (40,0\%) no Paraná e 7 (17,5\%) no Rio Grande do Sul. Além de identificar quantas e quais disciplinas poderiam abordar o conteúdo de Estatística ou Educação Estatística, verificou-se que os cursos se distribuem da seguinte forma: $16(44,4 \%)$ têm uma disciplina, $18(50,0 \%)$ têm duas e $2(5,6 \%)$ têm três, totalizando 59 disciplinas distribuídas nos 36 cursos analisados. O conjunto das disciplinas em cada curso, em média, tem carga horária de 105 horas ( $D P=31$ horas).

Nos dois trabalhos descritos, os pesquisadores evidenciaram a necessidade de olhar para os Projetos Pedagógicos de Cursos, a fim de elucidar o oferecimento da disciplina e o seu real desenvolvimento nos cursos de licenciaturas. Seguindo essa mesma perspectiva, Batanero (2002) aduz que o fato de conteúdos estatísticos fazerem parte dos currículos 
oficiais não significa que sejam ensinados nos diversos níveis escolares, pois, paralelamente às questões curriculares, surgem as questões de formação dos professores (CONTI; NUNES; GOULART, 2018).

Complementando isso, Costa e Nacarato (2011) afirmam que,

O ensino da Estatística, nos cursos de licenciatura, fica atrelado ao formador que, muitas vezes, tem dificuldade em lidar com esses conteúdos que devem estar presentes na licenciatura e no bacharelado. A Estatística apresentada na licenciatura, com frequência, não é capaz de dar subsídios aos professores para atuar nas salas de aula, exigindo que busquem em cursos de formação continuada a capacitação para trabalhar a estocástica em sala de aula. (COSTA; NACARATO, 2011, p. 388)

Nessa mesma perspectiva, Costa e Pamplona (2011, p. 899) complementam que "a atual conjuntura aponta a necessidade de transformação da Licenciatura, de modo que ela possa oferecer referências a partir das quais os estudantes dela egressos, de fato, estruturem suas práticas de Educação Estatística na Educação Básica".

Com investigações relacionadas à sala de aula invertida no ensino de Educação Estatística, tivemos dois trabalhos: Costa, Costa e Carvalho (2019) e Andrade, Almeida e Esquincalha (2019).

No primeiro, os autores (2019) analisaram as possibilidades da metodologia ativa "sala de aula invertida", com o uso do Facebook como Ambiente Virtual de Aprendizagem, utilizado por alunos do Ensino Superior da Universidade do Estado do Mato Grosso, na disciplina de Estatística. Os sujeitos da pesquisa foram alunos da 3a fase do curso de Administração, do campus de Juara-MT. A partir das análises da observação participante, a entrevista e os registros audiovisuais indicaram que o uso do Facebook, para a sala de aula invertida, possibilitou ao professor desenvolver atividades de aprendizagem interativa, em grupo, na sala de aula. E, fora da sala de aula, dar orientações aos alunos baseadas em tecnologias digitais, tendo como característica marcante o melhor aproveitamento do tempo presencial com os alunos, evitando o desperdício desse tempo com aulas expositivas, muitas vezes menos produtivas do que aquelas propiciadas pelas interações online.

No segundo trabalho desenvolvido por Andrade, Almeida e Esquincalha (2019), foram analisadas as possibilidades do uso da sala de aula invertida na abordagem de ensino da Estatística no Ensino Superior, realizada com alunos da disciplina de Estatística dos cursos de Engenharia Mecânica e de Produção de uma instituição pública do Rio de Janeiro. Com os dados produzidos a partir dos textos postados pelos alunos na atividade em negrito no Quadro 1, relacionada ao feedback da disciplina, de um diário de campo elaborado pela professora e da observação da interação dos alunos no mural do Edmodo, concluíram que as atividades desenvolvidas possibilitaram tanto o desenvolvimento de uma atitude proativa, na qual os estudantes relataram facilidade de aprendizagem, como o desenvolvimento de uma rotina de estudos semanal.

A última tendência, presente nas discussões relacionadas as pesquisas sobre Educação Estatística no Ensino Superior, abrangeu as estratégias pedagógicas apresentadas com base em diferentes propostas. Em uma delas, Pereira (2019) relatou uma experiência de ensino de Estatística na área de ciências agrárias que buscou promover a aquisição das competências estatísticas em uma turma regular do curso de Zootecnia, da Universidade Tecnológica Federal do Paraná-Campus Dois Vizinhos. A estratégia pedagógica foi desenvolvida em 3 
(etapas): Pesquisa: os acadêmicos, em grupos de 3 a 5, foram instigados a realizar a coleta de dados de um assunto da área de Zootecnia e realizar o tratamento dos dados; Socialização: os acadêmicos apresentaram para os outros colegas da turma a pesquisa realizada; Avaliação: os acadêmicos avaliaram a atividade respondendo à pergunta: Quais as contribuições, desafios, limitações e dificuldades que esta atividade trouxe para a aprendizagem de Estatística? Os resultados apontados pelo autor destacam que as competências estatísticas foram desenvolvidas durante a atividade proposta e se efetivaram na apresentação de resultados de uma pesquisa, que envolveu assuntos do contexto profissional de um zootecnista. Corroborando essa assertiva, Campos, Wodewotzki e Jacobini (2011) argumentam que as estratégias pedagógicas utilizadas na Educação Estatística fundamentam-se na organização e desenvolvimento curricular, em que o aluno é o centro, tornando-se protagonista de sua aprendizagem, conforme apontados nos resultados de Pereira (2019). Dessa maneira, os alunos devem ser estimulados a se envolver no processo de produzir, organizar, refletir e discutir os dados e, posteriormente, construir e interpretar gráficos e tabelas (CAMPOS; WODEWOTZKI; JACOBINI, 2011).

Diante dos trabalhos elencados nesta temática, sistematizados no Quadro 2, pode-se indicar a relevância atribuída pelos pesquisadores à avaliação (JÚNIOR; ZAMORA; SOUZA, 2018), assim como ao contexto colaborativo (SCHREIBER; PORCIÚNCULA, 2019), à modelagem matemática (CAMPOS; COUTINHO, 2018; PERIM; WODEWOTZKI, 2018), ao currículo (ROCHA et al, 2019; CONTI; NUNES; GOULART, 2018), à sala de aula invertida (COSTA; COSTA; CARVALHO, 2019; ANDRADE; ALMEIDA; ESQUINCALHA, 2019) e às estratégias pedagógicas (Pereira, 2019).

Quadro 2 - Síntese dos trabalhos relacionados as tendências sobre Educação Estatística

\begin{tabular}{|c|c|c|c|}
\hline Temáticas & Autor(es) & Objetivo do trabalho & Principais resultados \\
\hline Avalição & $\begin{array}{l}\text { Júnior, Zamora e } \\
\text { Souza (2018) }\end{array}$ & $\begin{array}{l}\text { Investigar a relação entre a atitude e o } \\
\text { desempenho acadêmico de } \\
\text { estudantes do curso de Bacharelado } \\
\text { em Ciência e Tecnologia voltado para } \\
\text { conteúdo probabilístico e estatístico. }\end{array}$ & $\begin{array}{l}\text { Os alunos não consideram que as } \\
\text { listas de atividades dos } \\
\text { conteúdos de Probabilidade e } \\
\text { Estatística trazem benefícios para } \\
\text { o seu aprendizado. Os alunos } \\
\text { apresentam motivação inicial e } \\
\text { este fato gera melhores } \\
\text { resultados na primeira avaliação } \\
\text { da disciplina que versa sobre } \\
\text { conceitos básicos de } \\
\text { Combinatória e Probabilidade. }\end{array}$ \\
\hline $\begin{array}{c}\text { Contexto } \\
\text { colaborativo }\end{array}$ & $\begin{array}{l}\text { Schreiber e } \\
\text { Porciúncula (2019) }\end{array}$ & 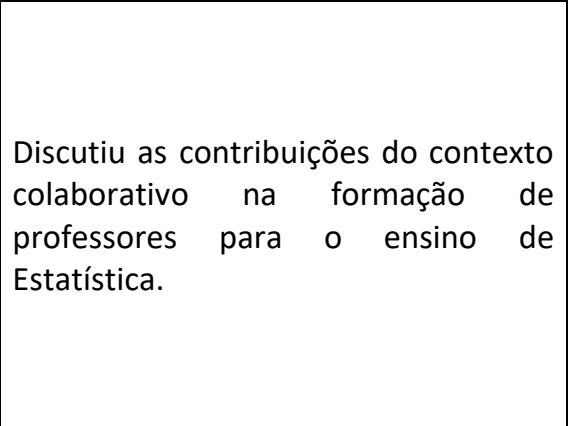 & $\begin{array}{l}\text { Como resultados da pesquisa, } \\
\text { pode-se indicar um interesse dos } \\
\text { professores no desenvolvimento } \\
\text { de projetos de pesquisas, em } \\
\text { especial, os Projetos de } \\
\text { Aprendizagem, possibilitando o } \\
\text { questionamento de suas práticas } \\
\text { pedagógicas e indicando } \\
\text { reflexões dos próprios fazeres } \\
\text { docentes. }\end{array}$ \\
\hline $\begin{array}{l}\text { Modelagem } \\
\text { matemática }\end{array}$ & $\begin{array}{l}\text { Campos e } \\
\text { Coutinho (2018) }\end{array}$ & $\begin{array}{l}\text { Apresentar um projeto de modelagem } \\
\text { matemática no qual os alunos da } \\
\text { disciplina de Estatística de um curso de } \\
\text { serviço em graduação são convidados }\end{array}$ & $\begin{array}{l}\text { Os resultados mostraram a } \\
\text { análise crítica por meio das } \\
\text { apresentações dos grupos que } \\
\text { apontaram erros nos gráficos dos }\end{array}$ \\
\hline
\end{tabular}




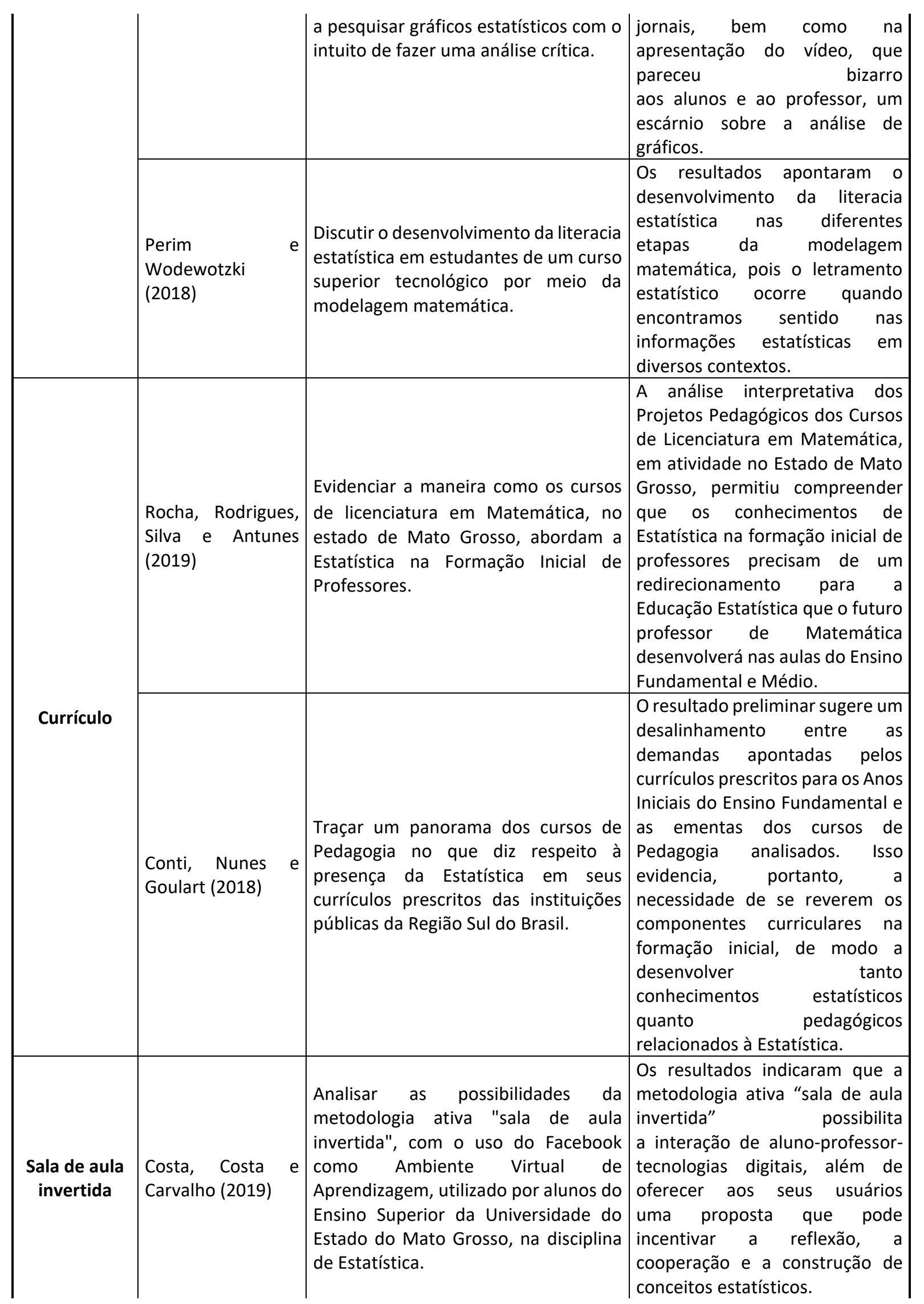




\begin{tabular}{|c|c|c|c|}
\hline & $\begin{array}{l}\text { Andrade, Almeida } \\
\text { e Esquincalha } \\
(2019)\end{array}$ & $\begin{array}{l}\text { Apresentar e discutir o resultado da } \\
\text { avaliação dos alunos depois da } \\
\text { realização de algumas atividades, } \\
\text { inspiradas em elementos da sala de } \\
\text { aula invertida, em uma disciplina de } \\
\text { Estatística dos cursos de Engenharia } \\
\text { de uma instituição pública do Rio de } \\
\text { Janeiro. }\end{array}$ & $\begin{array}{l}\text { Os resultados evidenciam que, } \\
\text { para além da melhoria na } \\
\text { aprendizagem e do envolvimento } \\
\text { e motivação dos estudantes, a } \\
\text { escolha, a flexibilização, o } \\
\text { desenvolvimento das atividades } \\
\text { e a utilização do ambiente virtual } \\
\text { Edmodo foram destacados como } \\
\text { pontos positivos, enquanto as } \\
\text { atividades práticas e o trabalho } \\
\text { em grupo foram classificados } \\
\text { como aspectos negativos. }\end{array}$ \\
\hline \multirow{3}{*}{$\begin{array}{c}\text { Estratégia } \\
\text { pedagógica }\end{array}$} & Silva e Samá (2019) & $\begin{array}{l}\text { Relatar a experiência no ensino e na } \\
\text { aprendizagem de Estatística durante } \\
\text { as aulas da disciplina de Estatística } \\
\text { Descritiva ministradas a estudantes do } \\
\text { curso de Bacharelado em } \\
\text { Biblioteconomia de uma Universidade } \\
\text { do Rio Grande do Sul. }\end{array}$ & $\begin{array}{l}\text { Os resultados mostraram o } \\
\text { potencial da criação de } \\
\text { ambientes de aprendizagem em } \\
\text { que os saberes es contextos dos } \\
\text { estudantes sejam legitimados, } \\
\text { quanto à importância do } \\
\text { trabalho coletivo e cooperativo } \\
\text { entre os mesmos no } \\
\text { compartilhamento de saberes e } \\
\text { no auxílio às aprendizagens dos } \\
\text { colegas. }\end{array}$ \\
\hline & Pereira (2019) & $\begin{array}{l}\text { Relatar uma experiência de ensino de } \\
\text { Estatística na área de ciências agrárias, } \\
\text { que buscou promover a aquisição das } \\
\text { competências estatísticas. }\end{array}$ & $\begin{array}{l}\text { Os resultados apontaram que os } \\
\text { acadêmicos, ao avaliarem a } \\
\text { atividade, deram votos de } \\
\text { positividade em sua totalidade, } \\
\text { em seus relatos, demonstraram } \\
\text { ter obtido um aprendizado com } \\
\text { significado, visualizando a } \\
\text { importância do estudo da } \\
\text { Estatística para sua atuação } \\
\text { profissional. }\end{array}$ \\
\hline & Figueiredo (2018) & $\begin{array}{l}\text { Analisar como as sequências de ensino } \\
\text { que envolvem conceitos de Estatística } \\
\text { básica podem contribuir na formação } \\
\text { docente de estudantes de Licenciatura } \\
\text { em Pedagogia }\end{array}$ & $\begin{array}{l}\text { As sequências que mobilizaram } \\
\text { conhecimentos relativos à } \\
\text { Estatística na Educação Básica } \\
\text { aplicadas pelos estudantes de } \\
\text { Licenciatura em Pedagogia } \\
\text { contribuíram para uma } \\
\text { aproximação da prática à teoria, } \\
\text { permitindo vislumbrar } \\
\text { possibilidades de reflexão sobre } \\
\text { a prática compartilhada entre } \\
\text { alunos. }\end{array}$ \\
\hline
\end{tabular}

Fonte: Dados da pesquisa

Neste caso, foram evidenciados, a partir da categoria C4, seis tendências atrelados à Educação Estatística, a saber: Avaliação, Contexto colaborativo, Modelagem matemática, Currículo, Sala de aula invertida e Estratégia pedagógica. Apontam indícios de que o processo de ensino e de aprendizagem no Ensino Superior, com enfoque na Educação Estatística, está alcançando diversos focos de estudos e cursos superiores, por exemplo: Matemática, Pedagogia, Zootecnia, Ciências Agrarias, Administração. A partir desta constatação interdisciplinar, estabelecemos um momento de reflexão: a Educação Estatística pode estar 
se apresentando como um "campo transversal de estudo", ou seja, deixou de atuar somente no campo da Matemática e está gradativamente perpassando por diversos cursos superiores, conforme os dados aqui levantados.

\section{Algumas considerações à guisa da conclusão}

Neste artigo, apresentou-se um Estado do Conhecimento da produção acadêmica sobre o ensino e aprendizagem de Educação Estatística no Ensino Superior. Para tanto, foram descritos e analisados 11 artigos, publicados na edição do XIII Encontro Nacional de Educação Matemática e do VII Seminário Internacional de Pesquisa em Educação Matemática.

A partir dos trabalhos analisados, é possível afirmar que o ensino e a aprendizagem de Educação Estatística no Ensino Superior presente nestes trabalhos focam a perspectiva da implementação de práticas pedagógicas estatísticas. Isso significa que se possibilita a articulação entre a teoria e prática dos fundamentos da Educação Estatística por meio de estratégias/atividades pedagógicas, sala de aula invertida fazendo uso de recursos tecnológicos, a modelagem matemática como metodologia para o desenvolvimento da Educação Estatística, pesquisas que discutiram a formação de professores em grupos colaborativos. Também foi discutido o currículo do curso de Licenciatura em Matemática e Pedagogia.

Como hipóteses operacionais que emergiram das análises, e que corroboram o que afirma Gil (2012), destacam-se as seguintes: (i) os cursos superiores de Licenciatura e/ou Bacharéis produzem conhecimento que efetivam, na prática, a Estatística e/eu Educação Estatística; e (ii) as pesquisas na área da Educação Estatística no Ensino Superior, que abordam a temática aqui discutida, precisam, ainda, ampliar as reflexões para responder às questões relacionadas aos conhecimentos relevantes à formação de professores que ensinam Matemática.

Por fim, espera-se que este Estado do Conhecimento possa contribuir não apenas para a compreensão dos processos de ensino e de aprendizagem da Educação Estatística no Ensino Superior, mas também para o desenvolvimento da Educação Estatística no cenário brasileiro, e que possa servir de base para futuras pesquisas tanto no âmbito das pesquisas científicas, como em sala de aula dos diferentes níveis de ensino. Em suma, um movimento de expansão e transformação dos temas já estudados no âmbito da Educação Estatística no Ensino Superior, revelados nos anais do ENEM/2019 e SIPEM/2018, indica ser necessário, num espaço mais amplo e articulado entre os pesquisadores e estudantes (KLÜBER, 2017).

\section{Referências}

ANDRADE, Fabiana Chagas de; ALMEIDA, Marcio Vieira de; ESQUINCALHA, Agnaldo da Conceição. Percepção de estudantes sobre uma abordagem para o ensino de estatística no contexto do ensino híbrido. In: ENCONTRO NACIONAL DE EDUCAÇÃO MATEMÁTICA, 13., 2019, Cuibá, MT. Anais... Mato Grosso, 2019. p. 1- 10.

BARDIN, Laurence. Análise de conteúdo. Tradução de Luís Antero Reto e Augusto Pinheiro. 3o reimp. da 1. ed. São Paulo: Edições 70, 2016.

BARDIN, Laurence. Análise de conteúdo. Lisboa: Edições 70, 1977. 
BATANERO, Carmen. Sentido estadístico: componentes y desarrollo. In: JORNADAS VIRTUALES EN DIDÁCTICA DE LA ESTADÍSTICA, PROBABILIDAD Y COMBINATORIA, 1., 2013, Granada (Espanha).

Actas... Granada: SEIEM, 2013. p. 55-61

BICUDO, Maria Aparecida Viggiani. Meta-análise: seu significado para a pesquisa qualitativa. Revista Eletrônica de Educação Matemática - REVEMAT, Florianópolis, Ed. temática, v. 9, p. 7-20, Jun. 2014.

BORBA, Rute Elizabete de Souza Rosa. O Raciocínio Combinatório na Educação Básica. In: ENCONTRO NACIONAL DE EDUCAÇÃO MATEMÁTICA. 10., 2010, Salvador. Anais... Salvador, 2010.p.1-16.

BRASIL. Ministério da Educação. Secretaria de Educação Fundamental. Parâmetros Curriculares Nacionais: Matemática. Primeiro e segundo ciclo do Ensino Fundamental. Brasília: MEC/SEF, 1997.

BRASIL. Ministério da Educação. Secretaria de Educação Fundamental. Parâmetros Curriculares Nacionais: Matemática. Terceiro e quarto ciclo do Ensino Fundamental. Brasília: MEC/SEF, 1998.

BRASIL. Ministério da Educação. Secretaria de Educação Média e Tecnológica. Parâmetros Curriculares Nacionais Ensino Médio: orientações educacionais complementares aos Parâmetros Curriculares Nacionais - Ciências da natureza. Matemática e suas tecnologias. Brasília: MEC/SEB, 2002.

BRASIL. Ministério da Educação. Secretaria de Educação Básica. Orientações curriculares para o Ensino Médio. Ciências da natureza, matemática e suas tecnologias. Brasília: MEC/SEB. 2006.

BRASIL. Ministério da Educação. Secretaria de Educação Básica. Base Nacional Comum Curricular. Brasília: MEC/SEF, 2018.

CAMPOS, Celso Ribeiro; COUTINHO, Cileda Queiroz e Silva. A modelagem matemática e o letramento estatístico no ensino de gráficos. In: SEMINÁRIO INTERNACIONAL DE PESQUISA EM EDUCAÇÃO MATEMÁTICA, 7., 2018, Foz do Iguaçu, PR. Anais... Paraná, 2018. p. 1- 10.

CAMPOS, Celso Ribeiro; WODEWOTZKI, Maria Lúcia Lorenzetti; JACOBINE, Otávio Roberto. Educação Estatística: teoria e prática em ambientes de modelagem matemática. Belo Horizonte: Autêntica Editora, 2011.

CAMPOS, Celso Ribeiro. Towards critical statistics education. Saarbrücken, Deutschland: Lambert Academic Publishing, 2016.

CAMPOS, Celso Ribeiro. A Educação Estatística: uma investigação acerca dos aspectos relevantes à didática da estatística em cursos de graduação. Tese (Doutorado em Educação Matemática). Instituto de Geociências e Ciências Exatas, UNESP-Rio Claro, 2007.

COSTA, Adriana.; NACARATO, Adair Mendes. A estocástica na formação do professor de matemática: percepções de professores e de formadores. Bolema, Rio Claro, v. 24, n. 39, p.367-386, nov. 2011.

COSTA, Wanderleya Nara Gonçalves.; PAMPLONA, Admur Severino. Entrecruzando fronteiras: a Educação Estatística na formação de professores de Matemática. Bolema, Rio Claro, v. 24, n. 40, p. 897-911. 2011. Edição Temática: Educação Estatística. 
CAZORLA, Irene Maurício. A relação entre a habilidade viso-pictórica e o domínio de conceitos estatístico na leitura de gráficos, 2002. Tese (Doutorado em Educação) Universidade Estadual de Campinas, Campinas.

CRESWELL, John W. Projeto de pesquisa: métodos qualitativo, quantitativo e misto. Tradução: Luciana de Oliveira da Rocha. 3. ed. Porto Alegre: Artmed, 2007.

CRESWELL, John. W. Projeto de Pesquisa: métodos qualitativo, quantitativo e misto. 3. ed. Tradução de Luciana de Oliveira da Rocha. Porto Alegre: Artmed, 2010.

CONTI, Keli Cristina; NUNES, Luciana Neves; GOULART, Amari. Desafios da educação estatística em cursos de pedagogia. In: SEMINÁRIO INTERNACIONAL DE PESQUISA EM EDUCAÇÃO MATEMÁTICA, 7., 2018, Foz do Iguaçu, PR. Anais... Paraná, 2018. p. 1- 10.

COSTA, Rosiane Moisés; COSTA, Rosicácia Florêncio; CARVALHO, José Wilson Pires. Sala de aula invertida no ensino de estatística: um estudo de caso no curso de Administração. In: ENCONTRO NACIONAL DE EDUCAÇÃO MATEMÁTICA, 13., 2019, Cuibá, MT. Anais... Mato Grosso, 2019. p. 1- 10.

DAMIN, Willian. A educação estatística e a formação de professores de matemática: contribuições de um projeto para a constituição dos saberes docentes. 2018. 148 f. Tese (Doutorado em Ensino de Ciência e Tecnologia) - Universidade Tecnológica Federal do Paraná, Ponta Grossa, 2018.

DENZIN, Norman K.; LINCOLN, Yvonna S. O planejamento da pesquisa qualitativa: teorias e abordagens. 2. ed. Tradução de Sandra Regina Netz. Porto Alegre: Artmed, 2006.

FERREIRA, Ana Cristina. O trabalho colaborativo como ferramenta e contexto para o desenvolvimento profissional: compartilhando experiências. In: NACARATO, Adair Mendes; PAIVA, Maria Auxiliadora Vilela. (Org.). A formação do professor que ensina Matemática. Belo Horizonte: Autêntica, 2013. p. 149-166.

FIGUEIREDO, Auriluci de Carvalho. Ensino de estatística: discussão sobre sequências didáticas aplicadas por estudantes de licenciatura em pedagogia em ambiente virtual. In: SEMINÁRIO INTERNACIONAL DE PESQUISA EM EDUCAÇÃO MATEMÁTICA, 7., 2018, Foz do Iguaçu, PR. Anais... Paraná, 2018. p. 1- 10.

GAL, Iddo. Adults' statistical literacy: meanings, components, responsibilities. International Statistical Review, Voorburg, v. 70, n. 1, p. 1-25, 2002. Disponível em: <https://iaseweb.org/documents/intstatreview/02.Gal.pdf>. Acesso em: 30 jan. 2020.

GATTI, Bernadete Angelina. A construção da pesquisa em educação no Brasil. Brasília: Plano, 2002.

GIL, Antônio Carlos. Como elaborar projetos de pesquisa. 4. ed. São Paulo: Atlas, 2002.

JÚNIOR, Ailton Paulo de Oliveira; ZAMORA, Pedro Rosental; SOUZA, Thiago Costa de. As atitudes em relação à probabilidade e à estatística e o desempenho acadêmico de alunos de alunos de uma bacharelado em ciências e tecnologia. In: SEMINÁRIO INTERNACIONAL DE PESQUISA EM EDUCAÇÃO MATEMÁTICA, 7., 2018, Foz do Iguaçu, PR. Anais... Paraná, 2018. p. 1- 10. 
KLÜBER, Tiago Emanuel; TAMBARUSSI, Carla Melli. A Pesquisa em Modelagem Matemática desde a VII Conferência Nacional sobre Modelagem na Educação Matemática - CNMEM. Revista Perspectiva em Educação Matemática, v. 10, n. 23, p. 563-583, 2017

\section{KLÜBER, Tiago Emanuel. Uma metacompreensão da Modelagem Matemática na Educação} Matemática. 2012. 396 p. Tese (Doutorado em Educação Científica e Tecnológica) - Universidade Federal de Santa Catarina. Florianópolis. 2012.

LOPES, Celi Espasandin. Educação estatística no curso de licenciatura em matemática. Bolema, Rio Claro, v. 27, n. 47, p. 901-915, dezembro de 2013.

LOPES, Celi Espasandin. O Ensino de Estatística e Probabilidade na Educação Básica e a Formação de Professores. Cad. Cedes. Campinas, vol. 28. n. 74. p. 57 - 73, jan. /abr. 2008.

MOROSINI; Marília Costa.; FERNANDES, Cleoni Maria Barboza. Estado do Conhecimento: conceitos, finalidades e interlocuções. Educação Por escrito, Porto Alegre, v. 5, n. 2, p. 154-164, jul./dez. 2014.

PENHA, Paulo César. A participação num grupo de dimensão colaborativa: reflexões sobre a minha prática docente. In: NACARATO, Adair Mendes; GRANDO, Regina Célia. (Org.). Estatística e

Probabilidade na Educação Básica. 1 ed. Campinas, SP: Mercado de Letras, 2013, p. 235-242.

PEREIRA, Luciana Boemer Cesar. Competências estatísticas e o ensino na área ciências agrárias: uma experiência no curso de Zootecnia. In: ENCONTRO NACIONAL DE EDUCAÇÃO MATEMÁTICA, 13., 2019, Cuibá, MT. Anais... Mato Grosso, 2019. p. 1- 10.

PERIM, Andréa Pavan; WODEWOTZKI, Maria Lúcia Lorenzetti. Contribuições da modelagem matemática para o desenvolvimento da literacia estatística: uma experiência em um curso superior tecnológico. In: SEMINÁRIO INTERNACIONAL DE PESQUISA EM EDUCAÇÃO MATEMÁTICA, 7., 2018, Foz do Iguaçu, PR. Anais... Paraná, 2018. p. 1- 10.

PORCIÚNCULA, Mauren; SAMÁ, Suzi. Projetos de Aprendizagem. In: PORCIÚNCULA, Mauren; SAMÁ, Suzi. (Org.). Educação Estatística: Ações e estratégias pedagógicas no Ensino Básico e Superior. Curitiba: CRV, 2015, p. 133-141.

ROCHA, Daniela Silveira et al. Estatística nas licenciaturas em matemática no estado de Mato Grosso. In: ENCONTRO NACIONAL DE EDUCAÇÃO MATEMÁTICA, 13., 2019, Cuibá, MT. Anais... Mato Grosso, 2019. p. 1-10.

SCARLASSARI, Nathalia Tornisiello.; LOPES, Celi Espasandin. Mapeamento dos trabalhos publicados nas seis primeiras edições do SIPEM pelo grupo de trabalho em educação estatística (GT12) da SBEM. REVEMAT, Florianópolis (SC), v.14, Edição Especial Educação Estatística, p.1-17, 2019.

SCHREIBER, Karla Priscila; PORCIÚNCULA, Mauren. Contribuições do contexto colaborativo na formação docente para o ensino de estatística. In: ENCONTRO NACIONAL DE EDUCAÇÃO MATEMÁTICA, 13., 2019, Cuibá, MT. Anais... Mato Grosso, 2019. p. 1-10.

SHULMAN, Lee $S$. Those who understand: knowledge growth in teaching. Educational Researcher, $v$. 15, n. 4, p. 4-14, 1986. Disponível em:

<http://www.fisica.uniud.it/URDF/masterDidSciUD/materiali/pdf/Shulman_1986.pdf>. Acesso em: 30 jan. 2020. 
SILVA, CAMILA RUBIRA; SAMÁ, SUZI. Experiências no ensinar e no promover o aprende estatística para estudantes do bacharelado em Biblioteconomia. In: ENCONTRO NACIONAL DE EDUCAÇÃO MATEMÁTICA, 13., 2019, Cuibá, MT. Anais... Mato Grosso, 2019. p. 1-10.

SILVA, Geferson Gustavo Wagner Mota da. Sala de aula invertida: uma abordagem no ensino de estatística. 2018. Trabalho de conclusão do curso de especialista em Ensino de Matemática no Ensino Médio, Universidade Federal de Santa Maria (UFSM, RS), 2018.

SOARES, Magdar Becker.; MACIEL, Fracisca Izabel Pereira. Alfabetização no Brasil: o estado do conhecimento. 2000. Disponível em http://www.mec.inep.gov. Acesso em: 29/01/2020.

VENDRAMINI, Claudete Maria Medeiros.; BRITO, Maria Regina Ferreira de. Relações entre atitude, conceito e utilidade da Estatística. Psicologia Escolar e Educacional. Perdizes (SP), v. 5 n. 1, p. 59-73, 2001.

VIALI, Lori. Aprender fazendo: como tirar proveito do computador para melhorar a aprendizagem da estatística. In: IX Encontro Nacional de Educação Matemática, 2007, Belo Horizonte (MG). Anais... Belo Horizonte, 2007. p. 1-15.

ZIEFFLER, Andrew, GARFIELD, Joan, FRY, Elizabeth. What Is Statistics Education? In: BEN-ZVI, D.; MAKAR, K.; GARFIEL, J. (ORGS) International Handbook of Research in Statistics Education. Gewerbestrasse: Springer International Handbooks of Education, 2018, p.37-71

WODEWOTZKI, Maria Lúcia L. JACOBINI, Otávio Roberto. O Ensino de Estatística no Contexto da Educação Matemática. In: BICUDO, M.A.V. \& BORBA, M. de C. (orgs.). Educação Matemática: Pesquisa em Movimento. São Paulo: Editora Cortez, 2004, p. 232-249. 